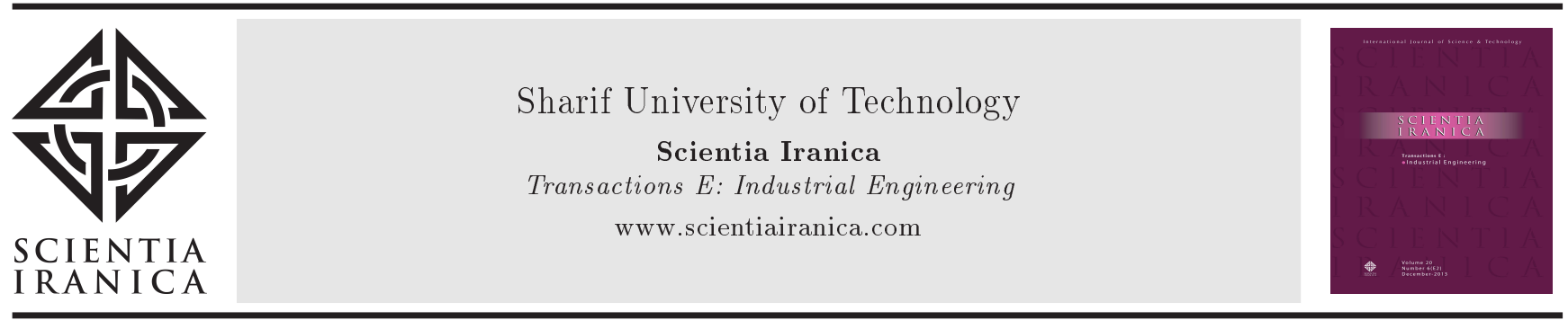

\title{
A new model to optimize the knowledge exchange in industrial cluster: A case study of Semnan plaster production industrial cluster
}

\author{
H.R. Dezfoulian, A. Afrazeh* and B. Karimi \\ Department of Industrial Engineering and Management Systems, Amirkabir University of Technology, Tehran, Iran.
}

Received 24 November 2015; received in revised form 9 January 2016; accepted 27 February 2016

\author{
KEYWORDS \\ Knowledge exchange; \\ Optimization; \\ Industrial cluster; \\ New mathematical \\ model; \\ Organizational social \\ relationships.
}

\begin{abstract}
Industrial clusters bring member firms the opportunities and advantages to save resources and increase competitiveness through cooperation and joint activities. One of these opportunities is knowledge exchange, using shared resources. If cluster firms want to create knowledge directly or acquire it out of cluster, it is necessary to spend much money and time. The aim is to maximize knowledge transfer between firms of a cluster regarding the limitation of budget and time, using existing knowledge flow networks. This problem is formulated with a new model of mixed integer programming and solved by the CPLEX solver for Semnan plaster production industrial clusters. The results of sensitivity analysis show that knowledge transfer is much more influenced by budget than by time constraints. The results help cluster managers to have a better understanding, regarding the available resources and business conditions, to maximize the results obtained from knowledge transfer process in industrial cluster members.
\end{abstract}

(C) 2017 Sharif University of Technology. All rights reserved.

\section{Introduction}

Knowledge is considered to be a critical resource to create competitive advantage among organizations [14]. Management and implementation of these resources are important activities in each organization. Creation, sharing, and using of knowledge effectively for innovation and sustaining competitive advantage are essential [5]. Knowledge management includes the process of identification, acquisition, development and creation, transfer, keeping, evaluation, and application of knowledge and helps organizations in achieving their objectives [6]. Huang [7] and Hsu [8] introduced knowledge transfer as a vital issue for organizations, since it empowers organizations to develop skills and compe-

*. Corresponding author. Tel.: +982164545364

E-mail address: Hrdezfoolian@aut.ac.ir (H.R. Dezfoulian); Afrazeh@aut.ac.ir(A.Afrazeh); B.karimi@aut.ac.ir (B. Karimi) tencies and competitive advantages $[9,10]$. Knowledge transfer is also considered as a requisite of successful knowledge management.

Knowledge transfer in intra-organizational and inter-organizational levels can be carried out [11-13]. The inter-organizational knowledge transfer can be done horizontally and vertically. The former takes place among the firms with similar grounds of production and activity so that they can belong to an industrial cluster and the latter usually occurs among the organizations existing in a supply chain in different levels [14]. Since the end of the 20th century, industrial cluster has been considered as the inflection point of industrial development programs and technology in most countries of the world. The industrial cluster is a network of firms with related industries that are located in an area [15-17]. These firms form relationships with each other to gain economic savings and have joint activities.

After the business atmosphere became more com- 
petitive, the competition from firm to firm changed into competition from cluster to cluster and chain to chain [18] and it became inevitable for firms to collaborate with each other to do joint activities to obtain success. Therefore, firms try to venture joint activities in integrated groups such as industrial clusters. One collaborative activity is knowledge transfer among cluster firms. Acquiring knowledge from outside or creating it within organization can be costly and time-consuming. The close relationship between the firms helps to facilitate the knowledge transfer and gain the knowledge from others more cheaply in a shorter length of time $[19,20]$. In addition to reduction in time and cost of knowledge acquiring, knowledge transfer enhances the synergy between the cluster members, improving the ability of cluster innovation and promoting the overall competitiveness of the cluster members [21].

There have been different studies about knowledge transfer in industrial clusters, but the academic research is limited to design and effective use of knowledge flow networks [22]. There has not been any special research on the design of effective networks of knowledge transfer in industrial clusters with optimization approaches. Design of effective network of knowledge transfer among the members of an industrial cluster based on the existing organizational relationships in the cluster leads to the facilitation of knowledge transfer and promotion of productivity of knowledge transfer in the industrial cluster. This, in turn, provides the ground to guide the managers to do effective actions. Although there is limited research about design of knowledge flow networks, the necessity of this subject demands more studies [22].

In this paper, our focus is on how it is possible to design effective knowledge transfer network based on the existing social networks between the members of a cluster, so that the level of knowledge among the cluster members is maximized regarding the constraint of budget and time. To gain the best result and improve productivity of knowledge transfer, the important types of knowledge are identified for cluster members and in transfer of knowledge between members, they are prioritized, because the value of knowledge is the most important factor in the transfer of knowledge [23,24]. Also, in knowledge transfer, degree of closeness must be paid attention to and firms with closer relationships are better candidates for knowledge transfer [25] as the knowledge transfer is carried out with lower expenses. Response to this question shows what knowledge in what time is transferred between which firms in an industrial cluster to maximize the knowledge level of cluster members regarding the constraint of budget and time. We formulated the problem in a new mixed integer programming model, solved it for a real problem, and presented some insights for managers to proceed with sensitivity analysis.
Therefore, important and influential parameters of this problem were identified and it became possible to make best decisions to exchange knowledge in cluster regarding the limitation of resources.

The rest of the paper will be presented as follows. The second section presents the literature review. Then, in the third section, the methodology is introduced and the definition of the problem and its mathematical formulation are presented in the fourth section. The fifth section is devoted to describing the case study and computational results. Finally, concluding remarks are summarized in the sixth section.

\section{Literature review}

Literature review of this research contains the previous research in two parts. The first part refers to the research on knowledge transfer and related issues in industrial clusters and the second part relates to organizational social relationship and its use in knowledge transfer.

\subsection{Knowledge transfer in industrial clusters}

There are a few studies about the transfer of knowledge in industrial clusters. Majority of studies about knowledge transfer in clusters are related to the methods and mechanisms of transfer. Sreckovic and Windsperger [26] examined the role of trust in using a knowledge transfer mechanism. Richardson [27] focused on knowledge sharing through social interaction in a policy-driven industrial cluster. Stacke et al. [28] analyzed knowledge transfer between clustered firms and its relation to the competitiveness of tourism destinations in southern Brazil. Sreckovic and Windsperger [29] argued that "tacitness of the partners' knowledge determines the information richness of the knowledge transfer mechanisms in clusters". Lopez-Saez et al. [30] assessed the applicability of the SECI model (Nonaka and Takeuchi) to the processes of external knowledge acquisition for firms located on knowledge-intensive clusters. Wilson and Spoehr [31] argued that informal knowledge transfers between skilled employees working in spatially bounded industrial clusters might have an association with the labour relationship between employers and employees.

The next group of studies examines the impact of various factors on knowledge transfer in industrial clusters. Hoffmann et al. [32] studied inter-firm cooperation, industrial support institutions, workforce mobility, and social ties in the Brazilian furniture cluster. Xiong et al. [33] utilized the theory of system dynamics to analyze the causal relationships of knowledge transfer in clusters. Four factors were incorporated in this model: the supplier of knowledge, the recipient of knowledge, the knowledge gap, and the transferring knowledge. Power and Lundmark [34] 
focused on the role of labor market and labor mobility as sources of knowledge and ideas in industrial clusters. Dayasindhu [35] studied the effect of knowledge transfer and tacit factors, such as trust, experience, opportunism, task complexity, and human asset specificity, on international competitiveness of industrial clusters.

Another group of studies deals with knowledge network in clusters. Guo and Guo [36] explored the evolution of knowledge network in manufacturing clusters considering the roles of participating actors in evolution stages of knowledge network. Chen et al. [37] studied the impact of the regional socio-cultural background, depth of the shared knowledge, extent of the shared knowledge, and attributes of knowledge, partners, and network on efficiency of knowledge networks in industrial clusters. Giuliani [38] explored the structural properties of knowledge networks in three industrial clusters by analyzing social networks.

Other papers have studied different subjects. Zhou [39] introduced a four-stage spiral cycle model for knowledge diffusion in high-tech clusters. Yu [40] studied product knowledge ontology modeling in the industrial cluster in order to solve the product information sharing problem. Hoffmann et al. [41] analyzed whether firm innovation was influenced by the transfer of knowledge among cluster firms. Fang et al. [21] took enterprise clusters as the research object in order to identify various risks during the process of knowledge sharing by use of fuzzy mathematics and artificial neural network. Bocquet and Mothe [42] turned to the government role in integration of knowledge in small industrial clusters.

The review of studies performed in this area shows that these studies are of quality type and pay attention to methods and factors influencing knowledge transfer in the cluster. The increasing competition in business and resource limitation necessitate that we do not limit ourselves to identify and survey the effect of factors influential in knowledge transfer. Therefore, it is necessary to determine the change and proper combination of these factors along each other to get the best result. The realization of this goal is possible by formulating the problem in mathematical optimization model.

\subsection{Organizational social relationships and their use in knowledge transfer}

Previous studies in knowledge management show that power of organizational social relationships affects the productivity of knowledge transfer remarkably [43-45]. In these studies, there are two relationships identified, which are used in knowledge transfer: strong and weak relationships [46,47]. Hansen proposes a seven-level scale to determine the power of relationships between organizations [48]. Using a scale of greater degrees can be useful in using the resources better. To determine the power of relationships between firms, the factors influencing them must be known.

The studies of factors influencing the formation of social ties are growing [22]. In these studies, different factors such as trust, resource exchange, communication method, frequent communications, and physical closeness are known to be effective in formation and reinforcement of social ties between organizations and the staff $[28,49]$. In this paper, we focused on factors such as financial relationships between firms and their levels, labor force, raw materials and equipment exchange [28], physical closeness [42], and family relationships between staff [50] to determine the power of relationships. The presence of strong relationship between firms increases the knowledge transfer efficiency [51] and reduces the cost [25]. On the other hand, researchers who have studied the social networks and organizations believe that firm success is dependent not only on talents of the staff and organizational capabilities, but also on their interactions [52].

Of the research carried out, which turned to the effect of social networks on promotion of knowledge sharing in organizations, some can be mentioned. Cowan and Jonard [53], using simulation, studied the effect of different types of network structures on knowledge distribution in organizations. Levine and Prietula [54], using agent-based simulation, studied the effect of different types of ties between workers on knowledge sharing. The results showed that the performative ties in organizations reduced the average time of finishing tasks. Dong et al. [55] proposed a model to integrate social network information, knowledge of employees, and availability of employees to benchmark managing performance of knowledge-intensive service organizations. They aimed to maximize the financial performance of organization for a given social network. The staff recognized the importance of using social network information and used it to facilitate knowledge sharing. In these studies, focus is on integrating features of staff and social network information and effective use of the available social network instead of optimum design and effective use of knowledge flow network.

More recently, research on designing organizational social network has developed to improve knowledge management in organizations [22]. Leung and Glissmann [56] designed the organizational social networks to improve skills of the staff. They used clustering approach to make connections among staff based on features and resources. In their clustering approach, there is no attention to the dynamic nature of staff's knowledge and the type of relationship among the staff (in terms of strength and weakness). Zhuge [57] introduced a frame to combine the organizational social relationships and capabilities of knowledge sharing in order to facilitate the design of a knowledge flow 
network. He used trial and error in this framework and did not maximize the total knowledge level of organization. Meanwhile, he did not pay attention to power of relationship amog the staff, knowledge transfer time, and cost constraints for knowledge transfer program. In another research, Dong et al. [22] studied the optimum design of knowledge flow networks among the staff and sought to maximize the total knowledge level of organization in their mathematical model.

In the above studies, while ignored among organizations, designing of social network among staff has been of interest. The closest research to ours is that of Dong et al. [22] who turned to optimum design of knowledge flow network among the staff using a mathematical model. They sought to maximize the knowledge level of staff in the objective function and did not consider the time and cost constraints for knowledge transfer in the organization. There are some constraints in real-life problems on time and cost, which most organizations face with. To have more attention to social ties between organizations that facilitate and reduce the knowledge exchange cost, in addition to maximization of knowledge level of cluster firms in the objective function, another section should be included to increase the knowledge transfer between firms that have stronger relationships with each other. Attention to firm power to acquire and transfer knowledge and capacity of training classes for simultaneous knowledge transfer to various firms is effective in making the model real. We tried to consider these issues in our proposed model to focus on real-life problems. Such a model can help us to better understand the optimum design of knowledge flow network between firms.

\section{Methodology}

This research is based upon the philosophy of positivism while it uses quantitative method and follows deductive approach. Evaluation of results, analysis of data and modeling, empirical data collection, generation of models, and development of instruments are some approaches of quantitative method. The strategy that is suitable for this study is survey, which is a technique in which secondary data is collected from documents of the firms. In this study, quantitative approach is preferred as an appropriate tool and all the results are presented in numbers and mixed integer programming model as well. Quantitative approach is a good fit for deductive approaches, in which a theory or hypothesis justifies the variables, and the purpose of quantitative research is imbuing the findings with larger populations through an objective [58]. The purpose of this study is to carry out a case study. Population and units of analysis are firms in Semnan plaster production industrial cluster and their panels of experts.

\section{Definition and formulation of the problem}

A number of firms, which are located in a geographic area with similar activities and products, have the potential of creating an industrial cluster. If these firms make strong relationships and create a network of cooperation, then an industrial cluster is formed. Member firms of an industrial cluster have the opportunity of increasing their efficiency by doing joint activities like supplying, using distribution networks, utilizing communications technology, staff training, doing research, and knowledge development. Knowledge transfer among members is another opportunity, which is the subject of this paper. It forms a knowledge network, which brings member firms the opportunity of jointly transferring knowledge with lower costs. Cluster managers can define an incentive system to form such knowledge networks. Their objective is maximizing member firms level of knowledge through increasing knowledge transfer in the shortest time and at the lowest cost.

Since the cluster member firms are not quite similar in terms of size, purpose, type of market, product features, etc., the importance of different types of knowledge is not the same for them. Also, various types of knowledge have not the same value for a firm. Therefore, prioritization of knowledge types is necessary when time and budget are limited. At the beginning of the planning horizon, it is necessary to determine the knowledge level of each firm in all types of knowledge. Three levels are defined for each type of knowledge: beginner, medium, and expert. The firms that have a higher knowledge level can transfer their knowledge to other firms in the process of knowledge transfer. The time of the transfer of knowledge is dependent on complexity and tacitness of knowledge.

A mathematical model is developed to maximize knowledge level of member firms with limited budget and time. This model is presented in the following. In the first part, the assumptions, notations, parameters, and variables of the model are described. Then, the mixed integer programming model for this problem is introduced.

\section{Assumptions}

There are some assumptions related to the problem:

- The cost of the transfer of knowledge is dependent on the type of knowledge and closeness of firms;

- Member firms have limited capability of transferring and acquiring knowledge simultaneously. This capability is dependent on attributes of the firm, such as the number of employees and their level of experience;

- Knowledge transferring firm is at higher level of knowledge than knowledge acquiring firm; 
- The firm that is transferring a kind of knowledge to others cannot simultaneously acquire the same knowledge from other firms;

- No firm can acquire a higher level of the knowledge that is being acquired until knowledge transferring period finishes;

- The level of knowledge for a firm increases when the knowledge transferring period finishes;

- The total cost of knowledge transfers cannot exceed the total budget allocated for the knowledge transfer in the industrial clusters.

\section{Notations}

$i, j, g \quad$ Firms belonging to cluster $1,2, \cdots, M$

$K \quad$ Types of knowledge $1,2, \cdots, K$

$T \quad$ Time periods $1,2, \cdots, T$

\section{Variables}

$X_{i j k}^{t} \quad$ If the transfer of knowledge $k$ from firm $i$ to firm $j$ starts in the period $t$, the value is one, otherwise zero

$E_{j k}^{t} \quad$ If the firm $j$ in the period $t$ is acquiring knowledge $k$, the value is one, otherwise it is zero

$F_{i k}^{t} \quad$ If the firm $i$ in the period $t$ is transferring knowledge $k$, the value is one, otherwise it is zero

$L_{i k}^{t} \quad$ Level of knowledge $k$ in the firm $i$ at the end of the period $t$ (a positive integer variable)

\section{Parameters}

$D_{k} \quad$ The number of periods required to transfer a level of knowledge $k$

$W_{i k} \quad$ Importance of knowledge $k$ for firm $i$

$C_{i j k} \quad$ Cost of transfer of knowledge $k$ from firm $i$ to firm $j$

$C \quad$ The total budget allocated for the knowledge transfer in the industrial clusters

$A_{i} \quad$ The maximum number of knowledge types the firm $i$ can transfer to other firms simultaneously (transfer capability of firm $i$ )

$B_{j} \quad$ The maximum number of knowledge types firm $j$ can acquire from other firms simultaneously (acquiring capability of firm $j$ )

$\theta \quad$ The maximum number of firms that can acquire a certain type of knowledge from a firm simultaneously (training class capacity in cluster)

$R_{i j} \quad$ Closeness of firm $i$ to firm $j$ $\alpha, \beta \quad$ Weight coefficients to determine the importance of each part of objective function, sum of which must be one

\subsection{Mathematical model}

Objective function and 14 sets of constraints of the model are the following.

\subsubsection{Objective function}

The objective function consists of two parts. The first part is to maximize the total knowledge level of industrial cluster firms regarding the knowledge values. The second part is to maximize the knowledge transfer between firms that have a closer relationship with each other.

$\max \left(\alpha \sum_{i=1}^{M} \sum_{k=1}^{K} W_{i k} \cdot L_{i k}^{T}+\beta \sum_{t=1}^{T} \sum_{i=1}^{M} \sum_{\substack{j=1 \\ i \neq j}}^{M} \sum_{k=1}^{K} R_{i j} \cdot X_{i j k}^{t}\right)$.

\subsubsection{Constraints}

The constraints of this model can be listed as follows:

- If the level of firm $i$ in knowledge $k$ is higher than firm $j$ at the beginning of the period $t$, then $X_{i j k}^{t}$ can be one ( $M^{\prime}$ is a large enough number):

$$
\begin{aligned}
& \left(1+M^{\prime}\right) \cdot X_{i j k}^{t} \leq L_{i k}^{t}-L_{j k}^{t}+M^{\prime} \\
& \quad i, j=1, \cdots, M, \quad i \neq j, \quad k=1, \cdots, K, \\
& \quad t<\left(T-D_{k}+1\right) .
\end{aligned}
$$

- If $X_{i j k}^{t}$ at the beginning of period $t$ is one, then firm $j$ cannot acquire knowledge $k$ from another firms in the next $D_{k}-1$ periods:

$$
\begin{aligned}
& \sum_{i=1, i \neq j}^{M} \sum_{q=t+1}^{t+D_{k}-1} X_{i j k}^{q} \leq\left(1-\sum_{i=1, i \neq j}^{M} X_{i j k}^{t}\right) \\
& j=1, \cdots, M, \quad k=1, \cdots, K, \\
& t \leq\left(T-D_{k}+1\right) .
\end{aligned}
$$

- In the last $D_{k}-1$ periods of planning horizon, the transfer of knowledge $k$ cannot be started, because there is not enough time to transfer it completely:

$$
\begin{aligned}
& \sum_{q=T-D_{k}+1}^{T} X_{i j k}^{q} \leq 0 \quad i, j=1, \cdots, M, \\
& i \neq j, \quad k=1, \cdots, K .
\end{aligned}
$$

- Knowledge level of firm $j$ remains the same for every type of knowledge in the initial $D_{k}$ periods of planning horizon:

$$
\begin{aligned}
& L_{j k}^{t+1}=L_{j k}^{t} \quad j=1, \cdots, M, \quad k=1, \cdots, K, \\
& t<D_{k} .
\end{aligned}
$$


- The knowledge $k$ of firm $j$ increases one level after completion of transfer period ( $D_{k}$ period):

$$
\begin{aligned}
L_{j k}^{t} & =L_{j k}^{t-1}+\sum_{i=1, i \neq j}^{M} X_{i j k}^{t-D_{k}} \quad j=1, \cdots, M, \\
k & =1, \cdots, K, \quad t>D_{k} .
\end{aligned}
$$

- The knowledge level $k$ of firm $i$ in all periods cannot be higher than the defined maximum level (expert level):

$$
\begin{aligned}
L_{i k}^{t} & \leq L_{\max } \quad i=1, \cdots, M, \quad k=1, \cdots, K \\
t & =1, \cdots, T
\end{aligned}
$$

- No more than $\theta$ firms can acquire knowledge $k$ from firm $i$ in period $t$ simultaneously:

$$
\begin{array}{cc}
\sum_{j=1, j \neq i}^{M} \sum_{q=t}^{t+D_{k}} X_{i j k}^{q} \leq \theta & i=1, \cdots, M \\
k=1, \cdots, K, & t=1, \cdots, T .
\end{array}
$$

- Constraints 9 and 10 control the capability of a firm in simultaneous knowledge transferring. Every firm $i$ can transfer maximum $A_{i}$ types of knowledge to other firms simultaneously in each period:

$$
\begin{gathered}
\sum_{k=1}^{K} F_{i k}^{t} \leq A_{i} \quad i=1, \cdots, M, \quad t=1, \cdots, T \\
\left(\sum_{j=1, j \neq i}^{M} \sum_{q=t-D_{k}+1}^{t} X_{i j k}^{q} / M^{\prime}\right) \leq F_{i k}^{t} \\
i=1, \cdots, M, \quad k=1, \cdots, K \\
t=1, \cdots, T .
\end{gathered}
$$

- Constraints 11 and 12 control the capability of a firm in simultaneous knowledge acquiring. Every firm $j$ can acquire maximum $B_{j}$ types of knowledge from other firms simultaneously in each period:

$$
\begin{aligned}
& \sum_{k=1}^{K} E_{j k}^{t} \leq B_{j} \quad j=1, \cdots, M, \quad t=1, \cdots, T \\
& \left(\sum_{i=1, i \neq j}^{M} \sum_{q=t-D_{k}+1}^{t} X_{i j k}^{q} / M^{\prime}\right) \leq E_{j k}^{t} \\
& j=1, \cdots, M, \quad k=1, \cdots, K \\
& t=1, \cdots, T .
\end{aligned}
$$

- Firm $j$ cannot acquire higher levels of knowledge $k$ from other firms as long as it receives it from firm $i$ :

$$
\begin{array}{ll}
\sum_{i=1, i \neq j}^{M} \sum_{q=t}^{t+D_{k}} X_{i j k}^{q} \leq 1 & j=1, \cdots, M, \\
k=1, \cdots, K, & t=1, \cdots, T .
\end{array}
$$

- Firm $i$ cannot acquire knowledge $k$ from other firms if it transfers the knowledge to firm $j$ :

$$
\begin{aligned}
& X_{i j k}^{t}+\sum_{g=1, g \neq i}^{M} \sum_{q=t}^{t+D_{k}-1} X_{g i k}^{q} \leq 1 \\
& i, j=1, \cdots, M, \quad i \neq j, \quad k=1, \cdots, K \\
& t=1, \cdots, T
\end{aligned}
$$

- The total costs of knowledge transfer from transferring firm $i$ to acquiring firm $j$ in planning horizon cannot exceed the allocated budget $C$ :

$$
\sum_{t=1}^{T} \sum_{i=1}^{M} \sum_{j=1, i \neq j}^{M} \sum_{k=1}^{K} C_{i j k} \cdot X_{i j k}^{t} \leq C .
$$

\section{Case study}

In this section, the proposed model for a real problem is applied and the results from the model and sensitivity analysis are presented while denoting the cluster characteristics. The studied industrial cluster is Semnan production plaster that has 35 industrial active firms in the production of plaster and its products. The distribution of firms is seen in Figure 1. Around 1000 and 4000 persons work in this cluster, directly and indirectly, respectively. Semnan, with an annual production of 6 million tonnes of plaster, produces $80 \%$ of the production of plaster in Iran. Averagely, 29 people are employed in each firm. At this stage, according to the idea of cluster managers, it is decided to incorporate 10 highly prioritized types of knowledge of clusters in the knowledge transfer program.

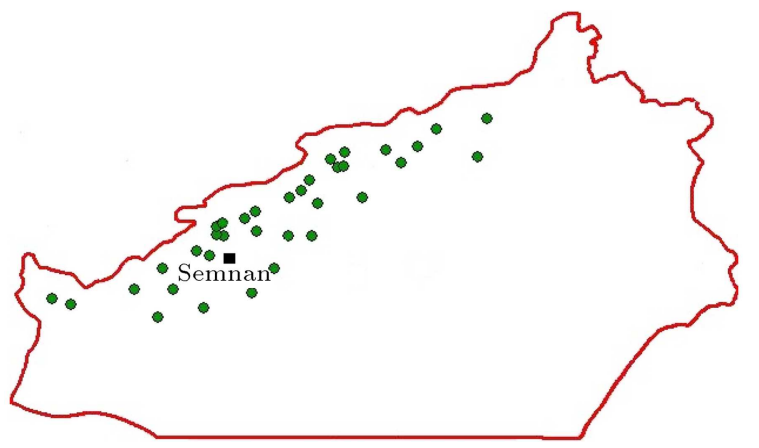

Figure 1. Distribution of plaster producing firms in the cluster. 
Initial knowledge level of member firms was identified for each type of knowledge at the beginning of the horizon as input parameters. This identification of initial knowledge level was based on the criteria defined by cluster experts and tailored to the performance of the firm in the field of knowledge.

More complex and tacit knowledge needs more time to be transferred between two firms. According to the view of experts, transfer of identified types of knowledge in this cluster takes 1 to 3 months, depending on their complexity and tacitness. Each month is considered a single period in this model.

The cost of knowledge transfer from one firm to another is related inversely to the closeness of the firms and directly to the complexity and tacitness of knowledge. In other words, the closer the relationship between the firms, the less the cost is; and the higher the complexity and tacitness of knowledge, the higher the cost of knowledge transfer would be [59]. The closeness of firms can be interpreted in terms of financial transactions between the firms, exchange of labor, raw materials and equipment, adjacency, and the relationship among their employees. The closeness of cluster members is expressed with numbers between 1 and 9 , where number 1 indicates the weakest relationship and number 9 represents the strongest relationship between the firms.

The capability of firms in simultaneous transferring or acquiring of knowledge is limited, and is dependent on the number of employees and their levels of education and experience. This capability varies between 2 and 6 types of knowledge for the firms, which are incorporated in this study.

Parameter $\theta$ depends on facilities of the cluster and possibility of coordinating firms to participate in the program. The value of $\theta$ in the Semnan plaster production industrial cluster was considered 4 .

The values $\alpha$ and $\beta$ are determined according to the view of cluster managers. They suggest values $\alpha$ and $\beta$ giving the importance of each objective function. In Table 1, values of parameters and the way of their measurement in the Semnan plaster production industrial cluster are presented.

The sum of levels of 10 highly prioritized knowledge types of member firms in the cluster, which are considered in the transfer process, is 714 at the beginning of the planning horizon and would be 1050 in the ideal conditions. It means that the maximum amount of knowledge that can be transferred is 336 .

Table 1. Summary of the model parameters.

\begin{tabular}{|c|c|c|c|c|}
\hline Parameters & Range/value & Average & Unit & Measurement and calculation basis \\
\hline$L_{i k}^{1 *}$ & $1-3$ & 2.04 & Level & $\begin{array}{l}\text { Performance of each firm in the knowledge of } \\
\text { interest based on the defined criteria }\end{array}$ \\
\hline$D_{k}$ & $1-3$ & - & Period (month) & Rate of complexity and tacitness of knowledge \\
\hline$W_{i k}$ & $0.03-0.19$ & 0.1 & - & Opinion of the managers of each firm \\
\hline$C_{i j k}$ & $11-49$ & - & Currency & $\begin{array}{l}\text { Rate of complexity and tacitness of knowledge } \\
\text { and closeness degree of firms to each other }\end{array}$ \\
\hline$C$ & 7500 & - & Currency & $\begin{array}{l}\text { Financial power of cluster members and the } \\
\text { budget allocated to cluster managers }\end{array}$ \\
\hline$R_{i j}$ & $1-9$ & 3.2033 & - & $\begin{array}{l}\text { Factors such as financial relationships between } \\
\text { firms and level of these relationships, exchange } \\
\text { rate of labour force, raw materials and } \\
\text { equipment, adjacency, and kin relationship among } \\
\text { staff of the firm }\end{array}$ \\
\hline$A_{i}$ & $2-6$ & 3.1428 & - & $\begin{array}{l}\text { Factors such as number of workers in the staff, } \\
\text { their education and experience level, facilities, } \\
\text { and the opportunity the firm considers for education }\end{array}$ \\
\hline$B_{j}$ & $2-6$ & 3.2571 & - & $\begin{array}{l}\text { Factors such as number of workers in the staff, } \\
\text { their education and experience level, facilities, } \\
\text { and the opportunity the firm considers for education }\end{array}$ \\
\hline$\theta$ & 4 & - & No. & $\begin{array}{l}\text { Available software and hardware facilities in the } \\
\text { cluster and the member firms for education }\end{array}$ \\
\hline$\alpha, \beta$ & $0.5-0.5$ & - & - & Opinion of the cluster managers \\
\hline
\end{tabular}

* The value $L_{i k}^{t}$ for $t=1$ is a parameter and for $t=2, \cdots, T$ is a variable. 


\subsection{Results}

The model is solved with the exact solution method and solver of CPLEX software of GAMS. The first part of the objective function is related to maximization of the firms' knowledge level. The results show that in a 13-month horizon, knowledge level of firms in the cluster, in 10 types, with cost of 7160 monetary units increases to the expert level (reaching ideal level of 1050 from the initial sum of 714). Limitation of resources does not allow the cluster managers to attain this level of knowledge transfer. Therefore, they can make decision about the selection of a combination of budget and time based on the results of sensitivity analysis and resources available for the best level of knowledge transfer. The second part of the objective function is related to enhancing the knowledge transfer between firms with stronger relationships. This reduces the cost of transferring knowledge between members of the cluster. Figure 2 represents the distribution of different levels of relationship between member firms. Relationship level 1 is the weakest relationship and relationship level 9 is the strongest relationship. While majority of relationships between member firms are weak (less than 5), knowledge transfers are mainly done through strong relationships (more than 5). This reflects that existing networks in cluster can be used to reduce the cost and facilitate the knowledge transfer.

Another important issue that should be noted is the role of the firms with expert and medium knowledge in the transfer of knowledge to other members of the cluster. The results indicated that $90.5 \%$ of the transferred knowledge in the cluster was from the firm that had experts in knowledge and the the share of firms with a medium knowledge in transfer was only $9.5 \%$. In Table 2, the share of transfer of knowledge between the firms according to their knowledge level is presented.

The contributions of firms to knowledge transfer program are not the same. As seen in Figure 3, 13 firms with less than 5 cases of knowledge transfer have the least contribution and 3 firms with more

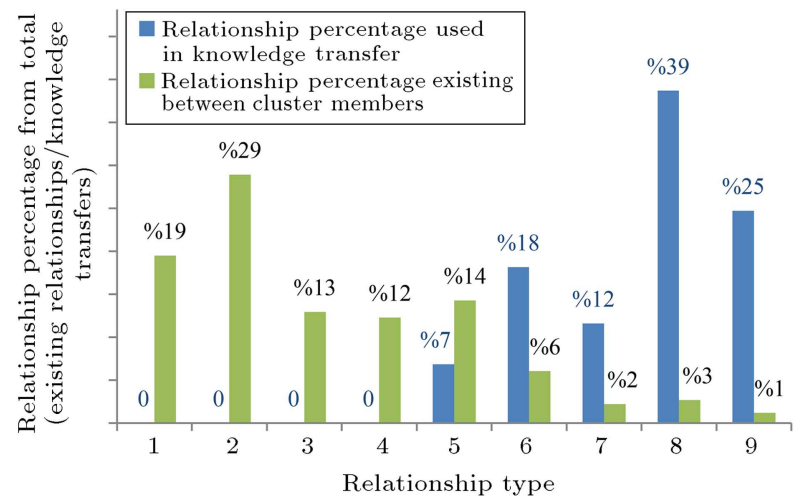

Figure 2. The existing relationships between the cluster members and relationship types used in knowledge transfer process.
Table 2. The share of firms in knowledge transfer regarding their knowledge level.

\begin{tabular}{ccc}
\hline $\begin{array}{c}\text { From medium } \\
\text { to beginner }\end{array}$ & $\begin{array}{c}\text { From expert } \\
\text { to medium }\end{array}$ & $\begin{array}{c}\text { From expert } \\
\text { to beginner }\end{array}$ \\
\hline $9.5 \%$ & $82 \%$ & $8.5 \%$ \\
\hline
\end{tabular}

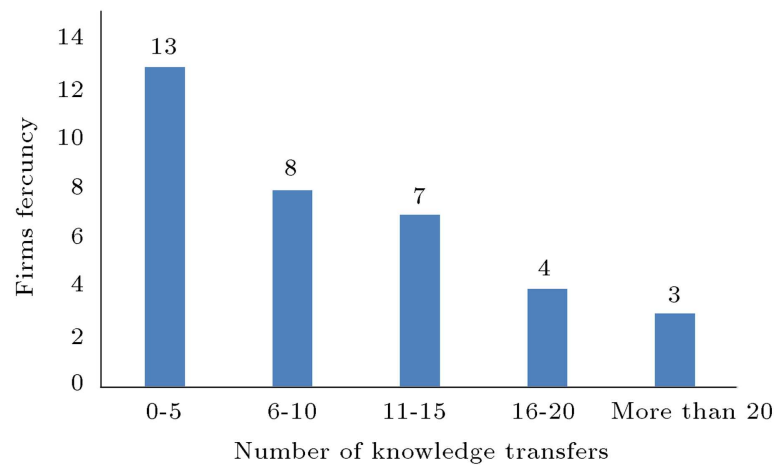

Figure 3. Firms frequency in terms of number of knowledge transfers.

than 20 cases of knowledge transfer have the greatest contribution to knowledge transfer. Close scrutiny of the input data of the model shows that mostly firms with closer relationships with other members and higher initial levels of knowledge have greater contributions to knowledge transfer to others.

The results of this section show that the firms which are expert in the knowledge of interest and have a close relationship with other members have the most influential role in the knowledge flow network of the cluster. Strength of network of firms in a cluster has prime importance in increasing the cluster potential for knowledge transfer.

In the next section, the results of examining the effects of model parameters on the final solution are presented so that the managers can choose the best combination of resources based on conditions and resources available.

\subsubsection{Sensitivity analysis of budget}

Without budget constraint, 336 transfers of knowledge occur and the ideal level of cluster knowledge (i.e., 1050) at a cost of 7313 monetary units is achievable. In the case of budget constraint, the number of knowledge transfers decreases. This relation between reduction of budget and decreasing number of knowledge transfers is shown in Figure 4, in which, percentages are defined with respect to the ideal point, i.e. allocation of 7313 monetary units and 336 knowledge transfers between members.

\subsubsection{Sensitivity analysis for planning horizon}

Achieving 336 knowledge transfers in the cluster requires a planning horizon of at least 13 periods. Shortening the length of planning horizon reduces the total number of knowledge transfers in the cluster, which 


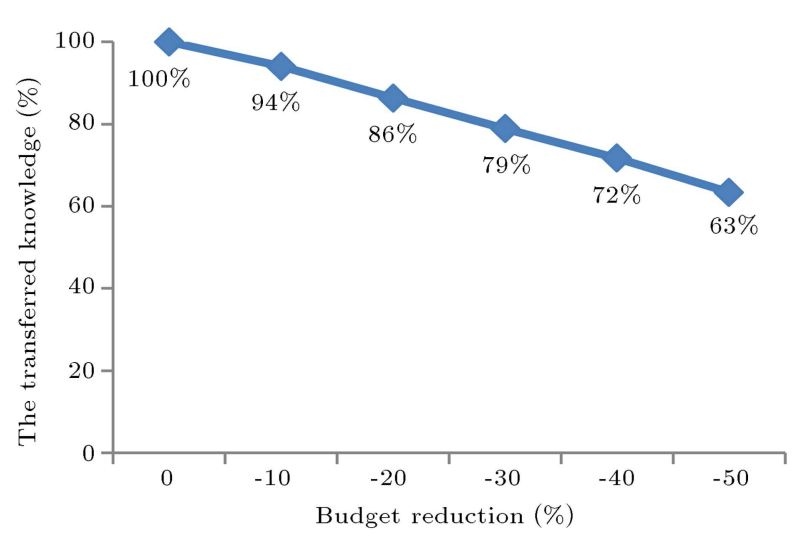

Figure 4. Sensitivity analysis of budget.

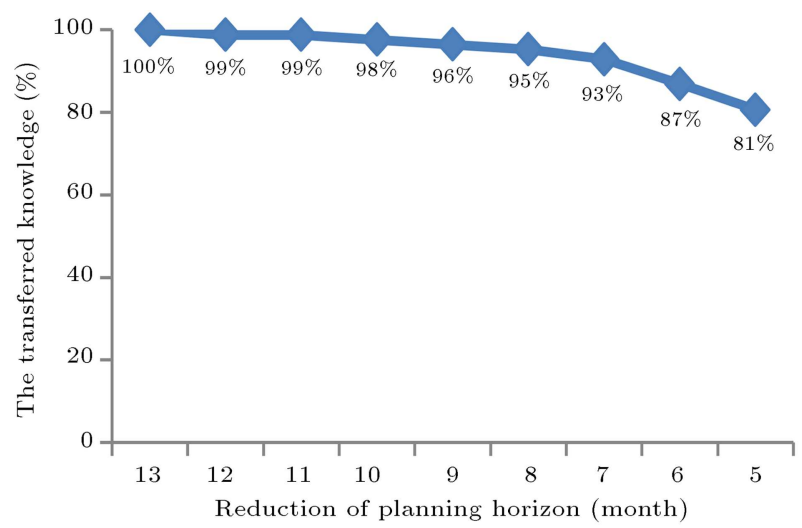

Figure 5. Sensitivity analysis for planning horizon.

in turn leads to reduction in the total level of cluster knowledge. Figure 5 shows the effect of reduction in the planning horizon on the total knowledge transfers in the cluster.

\subsubsection{Analysis of the relationship between training class capacity and knowledge transfer cost}

The presence of more representatives of firms in a class in order to acquire a type of knowledge is allowed if the facilities to increase the capacity of the classes are created. This requires spending part of the budget to increase the capacity of training classes in the firms. The increased capacity will help to reduce the cost and time of knowledge exchange; however, the results of sensitivity analysis in Figure 6 indicate that the change in capacity of trainnig classes from the present situation $(\theta=4)$ contributes up to $0.5 \%$ to the reduction in the cost of transfering knowledge, which can be completely ignored; in facet, it does not have any effect on the change of the knowledge transfer time (horizon of planning) and is neglected.

\subsubsection{Analysis of the relationship between the power of firms in simultaneous knowledge exchange and planning horizon}

Capacity of firms for simultaneous knowledge exchnge is an important factor in this model. This factor is

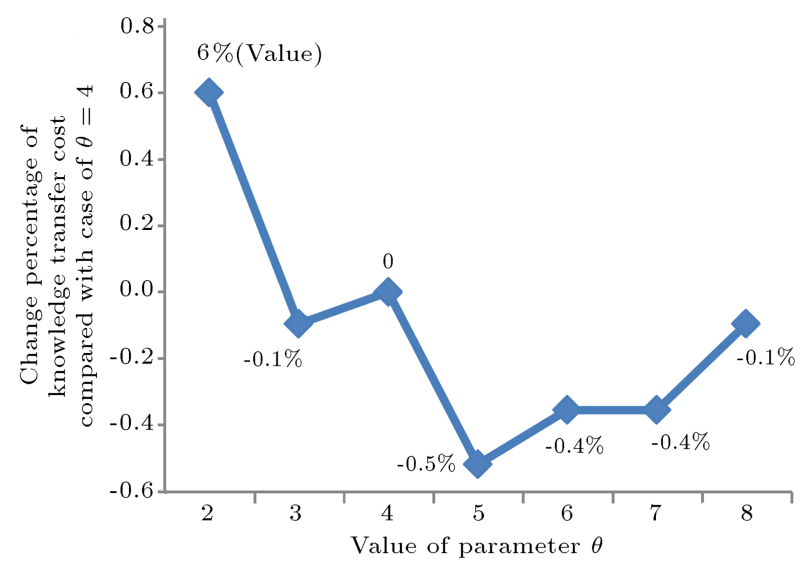

Figure 6. Relationship between training class capacity and knowledge transfer cost.

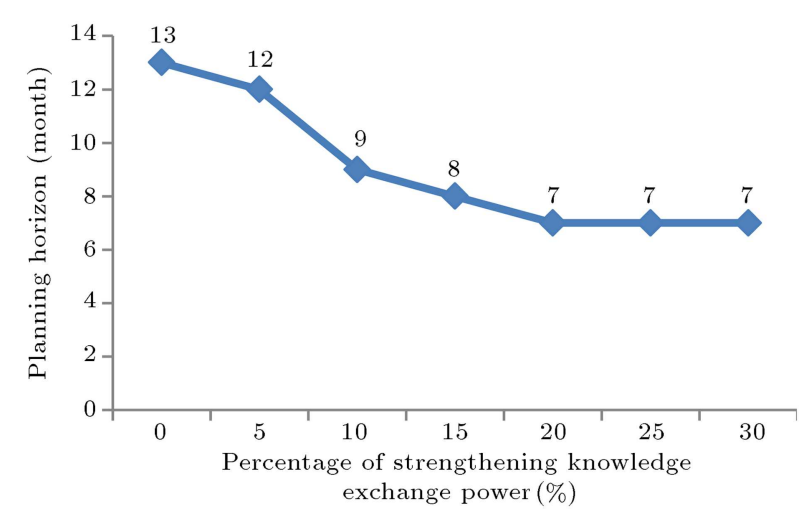

Figure 7. Relationship between strengthening the power of firms for simultaneous knowledge exchange and planning horizon.

entered into the model with parameters $A_{i}$ and $B_{j}$. Increase in these parameters does not affect knowledge transfer costs, but it may provide the possibility of reaching the ideal transfer number (336) in a shorter length of time (Figure 7 ). For example, $10 \%$ increase in power of knowledge exchnge in the firms makes transfer of 336 cases of knowledge become possible in 9 periods (4 periods fewer).

\subsubsection{Analysis of the relationship between closeness degree of the cluster members and knowledge transfer cost}

The quality of relationship between the firms is another influencing factor on the costs of knowledge transfer in industrial clusters. Strengthening these relationships brings the cluster managers the opportunity of reducing the costs. For example, in Figure 8, 25\% increase in strength of the relationships between the members causes $13 \%$ reduction in the costs of knowledge transfers.

\subsection{Discussion}

The status of cluster members' level of knowledge in the most important 10 types of knowledge at the beginning 


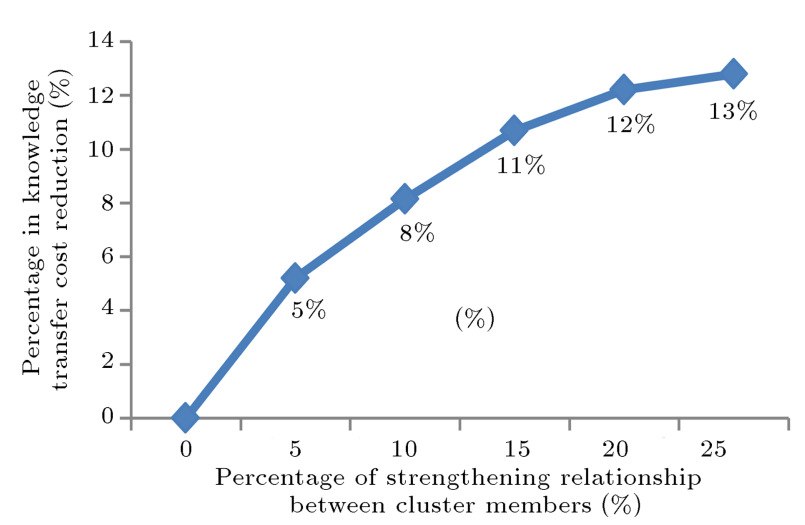

Figure 8. The relationship between strengthening closeness degree of the cluster members and knowledge transfer cost.

Table 3. Knowledge level of firms in the commencement of planning horizon.

\begin{tabular}{ccc}
\hline Beginner level & Medium level & Expert level \\
\hline $17.5 \%$ & $61 \%$ & $21.5 \%$ \\
\hline
\end{tabular}

of the horizon is presented in Table 3. Results of Table 3 show that expert-level firms are the most influential members for knowledge transfers and are in charge of $90 \%$ of the total knowledge transfers in the cluster. It is worth mentioning that an important part of the expert firms are those who were at medium level at first and reached the expert level through knowledge transfer process.

The strength of relationships among members of the cluster is an important factor that facilitates knowledge transfer process and reduces the costs. The results show that the knowledge transfer in the planning horizon occurs only between firms with strong relationships. This suggests utilizing the existing networks of the firms for the knowledge transfers.

Table 4 summarizes the impact of model parameters on cost and time of knowledge transfers. Changing parameters $\alpha$ and $\beta$ by determining the importance of parts of each objective function and the increase in the capacity of the training classes, i.e. parameter $\theta$, do not have a significant impact on cost and duration of the knowledge transfer in the cluster. Parameter $R_{i j}$ is an indicator of closeness of firms, and parameters $A_{i}$ and $B_{j}$ stand for the firm's power of simultaneous knowledge transfer that can reduce the cost and time, respectively. The results show that cluster managers can focus on strengthening the networks and increasing the capability of firms in absorbing and transferring knowledge to facilitate knowledge exchange with lower time and costs.

The sensitivity of the model to knowledge transfer budget is much more than time horizon, according to Figures 4 and 5 . This fact helps cluster managers in their decision-making and shows that budget cuts can strongly reduce the chance of implementing a successful knowledge transfer program.

\section{Conclusion}

The most important advantage of the formation of industrial clusters is joint activities of cluster members, which saves resources and facilitates synergy. An important instance is knowledge transfer using shared resources. With the identification of the influencing factors, we can design a cost-effective knowledge flow network, which maximizes knowledge transfer in a given time span. Accordingly, a mathematical model was developed in this paper for optimal design of knowledge flow network in industrial clusters. Then, the proposed model was applied in a real-world case study.

The results of solving the model revealed several important facts about the knowledge transfer between the member firms. First, expert members were key players in knowledge flow network and made a momentum, which accelerated knowledge transfers in the cluster. Therefore, the importance of these members should not be neglected in knowledge transfer program. Second, the process of knowledge transfer in the cluster depended heavily on knowledge transfer funding and budget cuts had a great impact on reducing the number of knowledge transfers among members. Third, increasing the capability of firms in knowledge absorption and transfer provided the opportunity of reducing time horizon of knowledge transfer program in industrial clusters. Fourth, strengthening the relationship between the firms significantly reduced the costs of knowledge transfer in the cluster. These facts help cluster managers to understand the most important factors in designing and implementing the knowledge

Table 4. Effect of model parameters on cost and time of knowledge transfer.

\begin{tabular}{lccc}
\hline \multicolumn{1}{c}{ Parameter } & $\begin{array}{c}\text { Knowledge } \\
\text { transfer cost }\end{array}$ & $\begin{array}{c}\text { Knowledge } \\
\text { transfer time }\end{array}$ & Efficiency \\
\hline Changes in $\alpha$ and $\beta$ & - & - & Ignorable \\
Increase of $\theta$ & - & - & Ignorable \\
Increase of $R_{i j}$ & Reduction & - & To $13 \%$ \\
Increase of $A_{i}$ and $B_{j}$ & - & Reduction & To $46 \%$ \\
\hline
\end{tabular}


transfer program in industrial clusters. They also can design knowledge flow network to maximize knowledge transfers in the cluster, with minimum cost and time.

In further research, the dynamic nature of relationships among cluster members should be considered as the interactions between cluster firms can cause them to become closer over time. The horizontal integration in supply chain is another issue to be studied in the development of the suggested model.

\section{References}

1. Jasimuddin, S.M. "Exploring knowledge transfer mechanisms: The case of a UK-based group within a high-tech global corporation", International Journal of Information Management, 27, pp. 294-300 (2007).

2. Narteh, B. "Knowledge transfer in developeddeveloping country interfirm collaborations: a conceptual framework", Journal of Knowledge Management, 12(1), pp. 78-91 (2008).

3. Hammami, H., Amara, N. and Landry, R. "Organizational climate and its influence on brokers' knowledge transfer activities: A structural equation modeling", International Journal of Information Management, 33, pp. 105-118 (2013).

4. Sheng, M.L., Chang, S.Y., Teo, T. and Lin, Y.F. "Knowledge barriers, knowledge transfer, and innovation competitive advantage in healthcare settings", Management Decision, 51(3), pp. 461-478 (2013).

5. Chamberlin, T. and Doutriaux, J. "Sourcing knowledge and innovation in a low-technology industry", Industry and Innovation, 17(5), pp. 487-510 (2010).

6. Afrazeh, A. "Knowledge management (concepts, models, measurement and implementation)", Amirkabir University of Technology, Tehran, Iran (2009) (In Persian).

7. Huang, Ch. "Knowledge sharing and group cohesiveness on performance: an empirical study of technology R\&D teams in Taiwan", Technovation, 29, pp. 786-797 (2009).

8. Hsu, C. "Knowledge sharing practices as a facilitating factor for improving organizational performance though human capital: Preliminary test", Export Systems with Applications, 35, pp. 1316-1326 (2008).

9. Ji, L-M., Hung, J., Chen, S-W. and Jou, C. "Fostering the determinants of knowledge sharing, virtual communities", Computers in Human Behavior, 25(4), pp. 929-939 (2009).

10. Wang, S. and Noe, R.A. "Knowledge sharing: A review and directions for future research", Human Resource Management Review, 20, pp. 115-131 (2010).

11. Fang, S.C., Yang, C.W. and Hsu, W.Y. "Interorganizational knowledge transfer: the perspective of knowledge governance", Journal of Knowledge Management, 17(6), pp. 943-957 (2013).
12. Hutzschenreuter, T. and Horstkotte, J. "Knowledge transfer to partners: a firm level perspective", Journal of Knowledge Management, 14(3), pp. 428-448 (2010).

13. Wijk, R.V., Jansen, J.J.P. and Lyles, M.A. "Interand intra-organizational knowledge transfer: A metaanalytic review and assessment of its antecedents and consequences", Journal of Management Studies, 45, pp. 830-853 (2008).

14. Kotabe, M., Martin, X. and Domoto, H. "Gaining from vertical partnerships: knowledge transfer, relationship duration, and supplier performance improvement in the US and Japanese automotive industries", Strategic Management Journal, 24, pp. 293-316 (2003).

15. Malmberg, A. and Maskell, P. "The elusive concept of localization economies: towards a knowledge based theory of spatial clustering", Environment and planning, 34(3), pp. 429-449 (2002).

16. Porter, M.E. "Clusters and the new economics of competition", Harvard Business Review, 76(6), pp. 7790 (1998).

17. Porter, M.E. "Location, competition, and economic development: local clusters in a global economy", Economic Development Quarterly, 14(1), pp. 15-34 (2000).

18. Hult, G.T.M., Ketchen, D.J. and Arrfelt, M. "Strategic supply chain management: Improving performance through a culture of competitiveness and knowledge development", Strategic Management Journal, 28, pp. 1035-1052 (2007).

19. Broekel, T. and Binder, M. "The regional dimension of knowledge transfers-A behavioral approach", Industry and Innovation, 14(2), pp. 151-175 (2007).

20. Kesidou, E. and Snijders, C. "External knowledge and innovation performance in clusters: Empirical evidence from the uruguay software cluster", Industry and Innovation, 19(5), pp. 437-457 (2012).

21. Fang, Y., Liang, Q. and Jia, Z. "Knowledge sharing risk warning of industry cluster: An engineering perspective", Systems Engineering Procedia, 2, pp. 412421 (2011).

22. Dong, S., Johar, M. and Kumar, R. "Understanding key issues in designing and using knowledge flow networks: An optimization-based managerial benchmarking approach", Decision Support Systems, 53, pp. 646-659 (2012-2).

23. Fatahi, K. and Afrazeh, A. "An integrated model for evaluating knowledge value in organizations", International Journal, WSEAS Transaction on Information Science and Applications, 2(2), pp. 138-143 (2005).

24. Liu, C. and Chen, S. "Determinants of knowledge sharing of e-learners", International Journal of Innovation and Learning, 2(4), pp. 434-445 (2005).

25. Fritsch, M. and Kauffeld-Monz, M. "The impact of network structure on knowledge transfer: an application of social network analysis in the context of regional 
innovation networks", The Annals of Regional Science, 44(1), pp. 21-38 (2010).

26. Sreckovic, M. and Windsperger, J., The Impact of Trust on the Choice of Knowledge Transfer Mechanisms in Clusters, Network Governance Contributions to Management Science, Springer-Verlag Berlin Heidelberg, pp. 73-85 (2013).

27. Richardson, C. "Knowledge-sharing through social interaction in a policy-driven industrial cluster", Journal of Entrepreneurship and Public Policy, 2(2), pp. 160177 (2013).

28. Stacke, A.R.N.P., Hoffmann, V.E. and Costa, H.A. "Knowledge transfer among clustered firms: a study of Brazil", Anatolia - An International Journal of Tourism and Hospitality Research, 23(1), pp. 90-106 (2012).

29. Sreckovic, M. and Windsperger, J. "Organization of knowledge transfer in clusters: a knowledge-based view", In New Developments in the Theory of Networks, Franchising, Alliances and Cooperatives, M. Tuunanen, J. Windsperger, G. Cliquet, G. Hendrikse, Eds., Springer, Berlin, pp. 318-334 (2011).

30. Lopez-Saez, P., Navas-Lopez, J.E., Martin-de-Castro, G. and Cruz-Gonzalez, J. "External knowledge acquisition processes in knowledge-intensive clusters", Journal of Knowledge Management, 14(5), pp. 690707 (2010).

31. Wilson, L. and Spoehr, J. "Labour relations and the transfer of knowledge in industrial clusters: why do skilled workers share knowledge with colleagues in other firmsin Industrial Clusters: Why do skilled workers share knowledge with colleagues ?", Geographical Research, 48(1), pp. 42-51 (2010).

32. Hoffmann, V.E., Lopes, G.S.C. and Medeiros, J.J. "Knowledge transfer among the small businesses of a Brazilian cluster", Journal of Business Research, 67, pp. 856-864 (2014).

33. Xiong, J., Duan, Z. and Wang, Y. "Modeling and simulation of the inter-organizational knowledge transfer impact factors in industrial clusters", The 19th International Conference on Industrial Engineering and Engineering Management, Springer-Verlag, Berlin, Heidelberg, pp. 161-171 (2013).

34. Power, D. and Lundmark, M. "Working through knowledge pools: Labor market dynamics, the transference of knowledge and ideas, and industrial clusters", Urban Studies, 41(5/6), pp. 1025-1044 (2004).

35. Dayasindhu, N. "Embeddedness, knowledge transfer, industry clusters and global competitiveness: a case study of the Indian software industry", Technovation, 22, pp. 551-560 (2002).

36. Guo, J. and Guo, B. "The evolution of knowledge network in manufacturing cluster: A case in China", Proceedings of the IEEE IEEM, pp. 890-894 (2008).

37. Chen, J., Chen, D. and Li, Z. "The analysis of knowledge network efficiency in industrial clusters",
International Seminar on Future Information Technology and Management Engineering, IEEE, pp. 257-260 (2008).

38. Giuliani, E. "The selective nature of knowledge networks in clusters: evidence from the wine industry", Journal of Economic Geography, 7, pp. 139-168 (2007).

39. Zhou, S. "Study of knowledge diffusion FSAI model for high-tech SMES clusters", The 19th International Conference on Industrial Engineering and Engineering Management, Springer-Verlag, Berlin, Heidelberg, pp. 783-794 (2013).

40. Yu, J. "Study on product knowledge ontology for industrial cluster", International Symposium on Knowledge Acquisition and Modeling, IEEE, pp. 108-112 (2008).

41. Hoffmann, V.E., Bandeira-de-Mello, R. and MolinaMorales, F.X. "Innovation and knowledge transfer in clustered interorganizational networks in Brazil", Latin American Business Review, 12, pp. 143-163 (2011).

42. Bocquet, R. and Mothe, C. "Knowledge governance within clusters: the case of small firms", Knowledge Management Research \& Practice, 8, pp. 229-239 (2010).

43. Afrazeh, A. and Zarinozv, I. "A conceptual framework for presenting and promoting human resource knowledge sharing in the supply chain as a social network promoting human resource knowledge sharing in the supply Chain as a social", International Journal of Services, Economics and Management (Special Issue on: Modeling and Analysis of Supply Chain Networks), 2(3/4), pp. 227-245 (2010).

44. Borgatti, S.P. and Cross, R. "A relational view of information seeking and learning in social networks", Management Science, 49(4), pp. 432-445 (2003).

45. Cross, R., Parker, A., Prusak, L. and Borgatti, S.P. "Knowing what we know: supporting knowledge creation and sharing in social networks", Organizational Dynamics, 30(2), pp. 100-120 (2001).

46. Guy, I., Jacovi, M., Shahar, E., Meshulam, N., Soroka, V. and Farrell, S. "Harvesting with SONAR - The value of aggregating social network information", In Online Social Networks, Proceeding of the TwentySixth Annual SIGCHI Conference on Human Factors in Computing Systems, M. Burnett, M. Costabile, T. Catarci, B. Ruyter, D. Tan, M. Czerwinski, and A. Lund, Eds., ACM Press, New York, pp. 1017-1026 (2008).

47. Levine, S.S. and Kurzban, R. "Explaining clustering in social networks: towards an evolutionary theory of cascading benefits", Managerial and Decision Economics, 27(2-3), pp. 173-187 (2006).

48. Hansen, M., Using the Wisdom of Others: Searching for and Transferring Knowledge, Presentation at the London Business School (1996).

49. Kotlarsky, J. and Oshri, I. "Social ties, knowledge sharing and successful collaboration in globally distributed 
system development projects", European Journal of Information Systems, 14(1), pp. 37-48 (2005).

50. Arregle, J.L., Hitt, M.A., Sirmon, D.G. and Very, P. "The development of organizational social capital: Attributes of family firms", Journal of Management Studies, 44(1), pp. 73-95 (2007).

51. Hansen, M.T. "The search-transfer problem: the role of weak ties in sharing knowledge across organization subunits", Administrative Science Quarterly, 44(1), pp. 82-111 (1999).

52. Hidalgo, C.A. "The value in the links: networks and the evolution of organizations", (2010). Available: http://www.chidalgo.org/Papers/Hidalgo_ SageChapter-.pdf.

53. Cowan, R. and Jonard, N. "Network structure and the diffusion of knowledge", Journal of Economic Dynamics and Control, 28, pp. 1557-1575 (2004).

54. Levine, S.S. and Prietula, M. "Towards a contingency theory of knowledge exchange in organizations", Academy of Management Best Paper Proceedings, Atlanta, GA (2006).

55. Dong, S., Johar, M. and Kumar, R. "A benchmarking model for management of knowledge-intensive service delivery networks", Journal of Management Information Systems, 28(3), pp. 127-160 (2012-1).

56. Leung, Y.T. and Glissmann, S.M. "A clustering approach to the design of knowledge-intensive service providers", (2011). Available: http://domino.research. ibm.com/library/cyberdig.nsf/papers/58C6B1D509E8 DCA68525780000603960.
57. Zhuge, H. "Knowledge flow network planning and simulation", Decision Support Systems, 42, pp. 571592 (2006).

58. Borrego, M., Douglas, E.P. and Amelink, C.T. "Quantitative, qualitative, and mixed research methods in engineering education", Journal of Engineering Education, 98(1), pp. 53-66 (2009).

59. Leppala, S. "An epistemological perspective on knowledge transfers: From tacitness to capability and reliability", Industry and Innovation, 19(8), pp. 631-647 (2012).

\section{Biographies}

Hamid Reza Dezfoulian is a $\mathrm{PhD}$ student in the Department of Industrial Engineering at Amirkabir University of Technology and Lecturer in the Department of Industrial Engineering at Bu-Ali Sina University. His main research interest lies in the field of knowledge management and industrial plant planning.

Abbas Afrazeh is currently Associate Professor of Industrial Engineering at Amirkabir University of Technology, Tehran, Iran. His main research interest lies in the field of knowledge management and human resource management.

Behrooz Karimi is currently Professor of Industrial Engineering at Amirkabir University of Technology, Tehran, Iran. His main research interest lies in the field of supply chain management and production planning. 